\title{
NATIONAL INSTITUTE OF OCEANOGRAPHY
}

WORMLEY, GODALMING, SURREY

\section{Conduction signalling in the sea}

Calculations on the impedance and return current distribution of an insulated conductor in the sea with earth return. by M. J. TUCKER

N.I.O. InTERnal Report No. A. 53 
NATIONAI INSTITUTE OF OCEANOGRAPHY

Worm1ey, Goda1ning, Surrey.

Conduction signalling in the sea

Calculations on the impedance and

return current distribution of an

insulated conductor in the sea

with earth return.

by

M.J. TUCKER

N.I.O. Internal Report No. A. 53

September 1971 


\section{CONTENTS}

Page No.

List of Symbols

Conduction signalling in the sea

Introduction

The distribution of electric current in the sea

The effects of a short wire

and of boundaries

Summaxy of conclusions

Appendix I The theory of the return current distribution

fippendix II The impedance of the wire

Figures 1 to 4

$11-14$

\section{FIGURE CAPTIONS}

Figure 1 Geometry for the calculations.

Figure 2 Characteristic length as a

function of frequency assuming

$$
\sigma=4 \Omega^{-1} \mathrm{~m}^{-1}
$$

Figure 3 The Kelvin Functions ker $x$ and kei $x$

Figure 4 A function proportional to the modulus of the total current in an annulus of unit radial increment.

NOTE

These calculations were made for a variety of reasons and it seemed worth while putting them all on record in the sane report.

The main section on conduction signalling has been prepared for presentation at a joint meeting of the Society for Underwater $T_{e}$ chnology and the Institution of Electronic and Radio Engineers.

The author wishes to thank his colleague Mr. J. Crease for his help with the Bessel Functions. 


\section{SYMBOLS}

Note: the S.I. system of units is used.

\begin{tabular}{|c|c|c|}
\hline$\sigma$ & $=$ & conductivity of sea water cohm $^{-1}$ metre $e^{-1}$ ) \\
\hline$i$ & $=$ & Electric current density (amp metre ${ }^{-2}$ ) \\
\hline $\mathbf{I}$ & $=$ & total electric current within a radius $r$ (amp) \\
\hline$I_{w}$ & $=$ & electric current in the conductor \\
\hline B & $=$ & magnetic flux density (weber metre ${ }^{-2}$ ) \\
\hline$r$ & $=$ & radial distance from the centre of the conductor \\
\hline $\mathbf{R}$ & $=$ & radius of the cable \\
\hline$\mu$ & $=$ & $\begin{array}{c}\text { permeability of sea water taken as } \mu_{0}=4 \pi \times 10^{-7} \\
\text { weber amp }{ }^{-1} \text { metre }\end{array}$ \\
\hline & $=$ & $2 \pi f=$ angular frequency of $I_{w}$ \\
\hline$x$ & $=$ & $(\mu \sigma \omega)^{\frac{1}{2}}$ is a parameter with dimensions of $L^{-1}$ \\
\hline 0 & $=$ & $\begin{array}{l}1 / k \text { is the characteristic length defining the scale } \\
\text { of the phenomenon }\end{array}$ \\
\hline$x$ & $=$ & $r / r_{0}=k r$ is a non-dimensional normalised radius \\
\hline & $=$ & $R / I_{0}=k R$ \\
\hline
\end{tabular}




\section{Concuction signalling in the sea}

Some notes on the electric field produced in the sea by a single-core conductor with an earth return.

\section{Introduction}

Various snippets of new have appeared in the last few years concerned with the use of conduction fields in the sea for communication and other purposes (for example, Anon, 1968, Braincon Corp., 1969 and Schultz, 1971). One name used for such devices is SEDAR (Submerged Electrode Detection And Ranging), but the communication uses seem to be the ones of most likely practical application so that I prefer the term Conduction Signalling.

Such systems operate by injecting electric current into the sea from a pair of electrodes, and detecting the resulting field at a distance by means of another pair of electrodes. A quick evaluation shows that one cannot neglect inductive effects even at frequencies of a few herz, and so it has to be regarded as an electro-magnetic propagation problem. However, an order-of-magnitude calculation shows that electric displacement currents can be neglected over the frequency range of irterest.

In 1921 Carson and Gilbert worked out the impedance of an electric cable in the sea with an earth return. They were concerned with the case of a cable with an earthed sheath and/or an earthed cage of armouring wires. This is a more complicated problem than the case of present interest, and though the results we require may be extracted from their paper, it may be of interest to give the derivation of the results used in our simpler problem, part1y because it illustrates some important conclusions which may get lost in the more complicated mathematics of the other. This derivation is contained in an appendix.

The distribution of the electric current in the sea.

The problem initia11y treated is that of a $10 \mathrm{ng}$, straight insulated cylindrical conductor in the sea with an earth return. It is somewhat similar to the well-known theory of the skin-effect in a cylindrical conductor, but turned inside out. There is, however, one important difference resulting from the fact that the currents in the sea are induced by a concentrated axial current in the conductor: no potential differences are produced in the water. At every point, the resistive back-e.m.f. is exactly balanced by the electromagnetically-induced e.m.f. The energy dissipated by the current flowing in the resistive sea water is supplied by a back-e.m. $f$. induced in the conductor by the changing magnetic field surrounding it. Thus, apart from end effects, there is no potential difference in the water between the electrodes at the two ends of the cable. One can easily see that this must be so. The results below show that as the radial distance from the conductor is increased, the current and magnetic fields fall to zero in a rapidy convergent manner. There are therefor no potential gradients in the water a long way from the wire. There are no sources of e.m.f. available to support radial potential gradients. Therefore there are no potential gradients close to the wire where the strongest currents are flowing. A pair of electrodes connected by a wire parallel to the primary conductor will, however, apparent1y pick up a voltage equal to 
the resistive potential drop of the current flowing at that radius. The voltage is actually produced by the magnetic field cutting the wire, and a consequence of this which could be important in some cases is that it will depend on the routing of the wire and not only on the position of the electrodes. However, for the rest of this paper we shall assume for convenience that the voltage developed by the receiver is equal to the resistive component.

The phenomena are governed by a characteristic length $r_{0}=(\mu \sigma \omega)^{-\frac{1}{2}}$ Putting in a typical value of $\sigma$ for sea water of $4 \Omega^{-1} \mathrm{~m}^{-1}$ gives $r_{0}=178 / \sqrt{f}$ metres (figure 2). If the overall radius $R$ of the cable is less than $0.02 \mathrm{r}_{0^{\circ}}$ which will be true in al1 cases of interest here, then to an accuracy of better than 1 part in $10^{3}$ the current density $i$ in the water at a radial distance of $I$ from the centre of the conductor is given by $\frac{i}{I_{w}}=-\frac{1}{2 \pi r_{0}^{2}}(\operatorname{sei} x-j \operatorname{trer} x)$

where $x=r / r_{0}$ and kei $x$ and ter $x$ are Kelvin Functions which are tabulated (for example, by Oiver, 1964).

This function is plotted in figure 3, which shows the distribution of in-phase and quadrature current near the cable in parametric form.

For uncerwater signalling we are concerned with longer distaces. When $x>1$, equation (1) becomes

$\frac{i}{I_{w}}=\frac{1}{r_{0}^{2} \sqrt{(4 \pi x)}} e^{-(x / \sqrt{2})}\left[\sin \left(-\frac{x}{\sqrt{2}}-\frac{\pi}{8}\right)+j \cos \left(-\frac{x}{\sqrt{2}}-\frac{\pi}{8}\right)\right]$ $\ldots(\omega)$

We are also not concerned with the phase of the signal, so that

$\left|\frac{i}{I_{w}}\right|=\frac{1}{r_{0}^{2} \sqrt{(4 \pi x)}} e^{-(x / \sqrt{2})}$

This equation is correct to better than $1 \%$ for values of $x>3.6$

We can now put in some figures to get a feel for the problem (assuming $\tau=4 \Omega^{-1} \mathrm{~m}^{-1}$ )

$$
\begin{aligned}
\text { At } 300 \mathrm{Fz}, \mathrm{r}_{0} & =10.3 \mathrm{~m} \\
\text { Voltage gradient } & =|i| / \sigma \simeq i / 4 \text { VoIts/netre } \\
& =\left|r_{\mathrm{r}}\right| \frac{2.24 \times 10^{-3}}{\sqrt{\mathrm{r}}} \mathrm{e}^{-\mathrm{r} / 1} .5 \mathrm{~V}_{\mathrm{i} i}^{-1}
\end{aligned}
$$


At a range of $100 \mathrm{~m}$, the voltage gradient is therefore

$$
\left|I_{w}\right| \times 2.2 \times 10^{-7} \mathrm{Vm}^{-1}
$$

Beyond this the gradient falls off by slightly more than $1 / e$ for every $14.5 \mathrm{~m}$.

One must remember that the impedance and bandwidths are very $10 \mathrm{w}$, so that an $I_{w}$ value of $100 \mathrm{~A}$ is probably attainable, and $10^{-8} \mathrm{v}$ can probably be detected. Even so, this formula would lead to the conclusion that the maximum range at $300 \mathrm{~Hz}$ is unlikely to exceed $200 \mathrm{~m}$.

Even at $30 \mathrm{~Hz}$, the voltage gradients at $500 \mathrm{~m}$ range are approximately $2.5 \times 10^{-10} \mathrm{I}_{\mathrm{w}} \mathrm{Vm}^{-1}$, which must be near the limit of detectability. The effects of short source wires and of boundaries

We have assumed so far that the primary wire is infinitely long. Equation 2 shows that we are dealing with propagating electromagnetic waves with a wavelength of $2 \sqrt{2} \pi r_{0}=8.88 r_{0}{ }^{\circ}$ This is, as one would expect, the wavelength calculated for plane e.m. waves (see, for example, Horvat, 1969). However, the attenuation length is only $\lambda / 2 \pi$ and so the beam-forming process will not work in the normal way. It might be possibie to calculate the patterns, but for the present discussion we need only make two elementary points concerning the effect of shorter wires.

(1) The field strengths will never be significantly above those calculated for an infinite source wire and will usually be less.

(2) They will genera1ly be greatest in a plane perpendicular to the wire and passing through its centre.

We have also assumed an infinite sea in al1 3 dimensions. The effect of a near-by surface is very problematical. Various authors quote measured ranges far in excess of those calculated by the above methods (for example, Anon. 1963. Horvat, 1969 quotes references to similar results with submerged dipoles, which is a closely related system). The explanation usually given for such results is that the radio waves "escape" into the air, travel as a surface wave, and reenter the water to reach the receiver. This is likely to be an effective mechanism only when the depth of the source plus the depth of the receiver is considerably less than the limiting range calculated for the all-sea path.

Sunmary of conc1usions

(1) If, as seems 1ikely, $30 \mathrm{~Hz}$ is the lowest practicable frequency at which to work such a system, the range of a deep system is Iinited to about 500 metres. At $300 \mathrm{~Hz}$ the maximum expected range is about $200 \mathrm{~m}$. At voice frequencies even lower ranges are expected and frequency equalisation ought to be difficult, though Schultz (1971) did not $f$ ind this a problem.

(2) The effect of short transmitter wires is to reduce the above ranges, but this reduction may not be great for wires of practical length (say 100m.). 
(3) The effect of shallow transmitters and receivers is problematical but experiments indicate that much longer ranges can be obtained, the radio waves probably travelling most of the distance as surface waves on the air-water interface. 


\section{APPENDIX I: the theory of the return current distribution}

Consider an insulated long straight concuctor of overall racius $R$ surrounded by an infinite volume of sea water. The following assumptions will be made:

(a) Axial symetry

(b) The displacement current tern in the electromagnetic equations may be neglected. In plane wave propagation in the sea this is valid for frequencies below about $10^{7} \mathrm{~Hz}$ (Horvat, 1969). It is equivalent to saying that the scale of the phenomenon with which we are dealing is sma11 compared to the wavelength in a medium with the sane permeability and dielectric constant but no conductivity. The results show this to be the case.

Note that $i$ and $I$ have been taken as positive in opposite directions. Though not perhaps formally correct, this avoids the currents in the water appearing with a negative sign.

Refer to figure 1 and consider e.m.f.'s round the loop maried with arrows. That due to the change in current density with radius is given by

$$
(1 / \sigma)[i+\delta r(\partial i / \partial r)]-i / \sigma=(i / \sigma) \delta r \cdot \partial i / \partial r
$$

That due to the changing magnetic field is

$\partial(B \delta r) / \partial t=\delta x(\partial B / \partial t)$

These must balance, so that

$\frac{1}{\sigma} \frac{\partial i}{\partial r}-\frac{\partial B}{\partial t}=0$

.

If the total electric current within the radius $r$ (incluting the current in the conductor) is $I$, then $B=\mu I / 2 \pi r$. Inserting this in equation (1) gives

$$
r \frac{\partial i}{\partial r}-\frac{\mu \sigma}{2 \pi} \frac{\partial t}{\partial t}=0
$$$$
\ldots
$$

Since $\partial I / \partial r=2 \pi r i$, one can differentiate equation (2) w.r.to $r$, substitute this equality, and with some reduction obtain

$$
\frac{\partial^{2} i}{\partial r}+\frac{1}{r} \quad \frac{\partial i}{\partial r}-\mu \sigma \frac{\partial i}{\partial t}=0 \quad \cdots \cdots
$$

which is the basic differential equation of the phenomenon

The temporal term is eliminated in the usual way by considering a sinusoidal current with angular frequency $w$, so that 
Substituting in equation (3) gives

$\frac{\delta^{2} f}{\delta r^{2}}+\frac{1}{r} \frac{\delta f}{\delta r}-\mu \sigma \cdot j \omega f=0$

Putting $\mu \sigma \omega=k^{2}$ and $x=k r$ gives

$x^{2} \frac{\delta^{2} f}{\delta x^{2}}+x \frac{\delta f}{\delta x}-j x^{2} f=0$

The solution of this is a Bessel function expressed in Kelvin functions (see, for example, Olver 1964, equations 9.9.3) and is

$f=c\left(\operatorname{ber} x+j \operatorname{bei} i_{0} x\right)+D\left(\operatorname{ker} x+j k e i_{0} x\right)$

Now ber $x$ and bei $x$ are functions increasing rapidly with $x$, whereas from physical reasoning we know that $f$ decreases as $x \rightarrow \infty$ therefore $\mathrm{C}=\mathrm{O}$ and (following the usual convention and omitting the subscript o)

$f=D(\operatorname{ker} x+j \operatorname{kei} x)$

ker $x$ and kei $x$ are tabulated (for examp1e, by O1ver 1964)

The value of the constant $D$ must now be determined, the criterion being that

$$
\begin{aligned}
\int_{R}^{\infty} 2 \pi \mathrm{r} i \mathrm{dr} & =I_{w} \\
\text { or } \mathrm{k}^{2} \mathrm{I}_{\mathrm{w}} / 2 \pi & =\int_{X}^{\infty} \mathrm{x} i \mathrm{dx} \text { where } \mathrm{X}=\mathrm{kR} \\
& =\int_{0}^{\infty} \mathrm{x} i \mathrm{dx}-\int_{0}^{X} \mathrm{x} i \mathrm{dx}
\end{aligned}
$$

Using equations (4) and (6)

$$
\begin{aligned}
\int_{0}^{x} x i d x & =\exp (j \omega t) D \int_{0}^{x}(x \operatorname{ker} x+j x k e i x) d x \\
& =\exp (j \omega t) D\left(x k e i x-j x k e x^{\prime} x\right)
\end{aligned}
$$

(O1ver 1964 equations 9.9.21)

kei' $x$ and knx $x$ both $\rightarrow O$ as $x \rightarrow \infty$, so equation (7) becomes:

$x^{2} I_{w} / 2 \pi=-\exp (j \omega t) \operatorname{DX}\left(\operatorname{kei}^{\prime} \mathrm{X}-\mathrm{j} \operatorname{kser}^{\prime} \mathrm{X}\right)$

or $D=-k^{2} I_{W} / 2 \pi x$ (kei' $X-j \operatorname{ker}^{\prime} X$ ) exp $j$ of

Using equations (4) and (6) gives

$\frac{i}{I_{w}}=\frac{k^{2}}{2 \pi x} \cdot \frac{1}{\operatorname{sei}^{\prime} x-j \operatorname{ker}^{\prime} x} \cdot(\operatorname{ker} x+j \operatorname{kei} x) \ldots$ 
In practice one is concerned with values of $x$ less than 0.1 , which represents the value for a cable $5 \mathrm{~cm}$ in diameter carrying $500 \mathrm{kHz}$ in water with a salinity of $35 \%$ at $15^{\circ} \mathrm{C}$. By considering the polynomial expansions of kei' $x$ and ker' $x$, and neglecting terms less than $10^{-3}$ of the modulus, one finds that

$$
\begin{aligned}
& \frac{1}{k e i^{\prime} x-\operatorname{ker} x}=\frac{\operatorname{kei} x+j \operatorname{ser}^{\prime} x}{(\operatorname{kej} x)^{\prime}+(\operatorname{ker} x)^{2}} \\
& \simeq-0.5 x^{3} \ln (x / 2)-\frac{j x}{1-0.393 x^{2}}
\end{aligned}
$$

When $x=0.1$ the real term here is less than $2 \%$ of the imaginary term and $0.393 x^{2}$ is less than $i \times 10^{-3}$, so for approxinate purposes this reduces to - j $x$. This is valid to better than 1 part in $10^{3}$ when $\mathrm{x}<0.02$. Using this approximation

$\frac{i}{I_{w}}=\frac{k^{2}}{2 \pi} \quad(k e i x-j k e r x)$

It is interesting to note that one can partially check this result very easily as follows. The total in-phase return current as a fraction of $I_{w}$ is

$$
\begin{aligned}
& =\int_{R}^{\infty} \text { real part of }\left(i / I_{w}\right) \cdot 2 \pi r d r \\
& =-k^{2} \int_{R}^{\infty} \text { rkei } x d x \\
& =-\int_{X}^{\infty} x \text { kei } x d x \\
& \simeq-\int_{0}^{\infty} x \operatorname{kei} x d x \\
& =1
\end{aligned}
$$

which is correct.

Similar1y,

$$
\begin{aligned}
& \int_{\mathbb{R}}^{\infty} \text { imaginary part of }\left(i / I_{w}\right) \cdot 2 \pi r d r \\
& \quad \therefore \int_{0}^{\infty} x \operatorname{ker} x d x \\
& \quad=0
\end{aligned}
$$




\section{APPENDIX II: The cable impedance}

First consider the cable to consist of a thin tube of perfectiy conducting material of radius $R$ separated from the water by a negligible thickness of insulation. The e.m.f. induced in this tube is then identical to that induced in the water immediately surrounding it. Since, as explained in the main part of the paper, in the water this is exactly balanced by the resistive voltage drop, it equals $-i_{R} / \sigma$ per unit length, where $i_{R}$ is the current at $r=R$.

$i$ and $I_{w}$ have been taken as positive in opposite directions, so a

further minus sign is required, and using equation (11) then gives the impedance per unit length as

$$
\begin{aligned}
z & =\frac{i_{R}}{\sigma I_{W}}=\frac{-k^{2}}{2 \pi \sigma} \quad(\operatorname{kei} x-j \operatorname{ker} x) \\
& =-\frac{\mu}{2 \pi} \quad s(\operatorname{kei} x-j \operatorname{ker} x)
\end{aligned}
$$

On the same assumptions as before, that is, that $x<0.025$, low value approximations for ker $X$ and kei $X$ can be used giving

$$
z=(\mu / 2 \pi) \omega[0.785-j(0.5772+\ln x / 2)] \quad \cdots
$$

Inserting $\mu=4 \pi \times 10^{-7}$ and using $n \mathrm{n}$ instead of $\mathrm{ln}(\mathrm{x} / 2)$ gives

$$
z=10^{-7} \omega[1.570+j(.2318-2 \cdot \ln x)] \Omega / m \ldots
$$

The impedance of a real single-eonductor cable can now be calculated in two stages. Firstly, one adds a hypothetical coaxial sheath of perfectly conducting material at the overall radius $R$ and passes the return current through this. The impedance of this hypothetical coaxial cable can be calculated by well-established methods. The impedance added by taking away the hypothetical sheath and substituting the water path is then that calculated above.

The fact that the resistive component is independent of cable diameter is easily explained by reference to figure 4 which shows that the power aissipation in the immediate vicinity of the cable is negligible. The fact that it is also independent of concuctivity was unexpected. It arises because the linear scale $\left(=r_{0}\right)$ of the current distribution is $\propto 1 / N \sigma$, so that the current $i$ per unit area close to the wire is $\propto \sigma$ and the refore the potential drop per unit length and hence the total power dissipation is independent of $\sigma$. It is in one respect a disappointing result. One of the reasons why the author worked through this theory was that he realised from physical reasoning that the resistive component would be largely independent of cable diamerer, and therefore hoped that measuring it would provide a means of determining conductivity in a manner insensitive to fouling.

Turning now to the reactive component, the first fact to note is that over the range of $X$ for which equation (14) is valid, $b n X$ is negative and large enough for the whole reactive term to be positive (that is, inductive) 
putting $X=R(\mu \sigma \omega)^{\frac{1}{2}}$, putting in the numerical value for $\mu$. and converting to logarithms to the base 10 gives

$L=\left[14.82-4.605 \log _{10} R-2.303 \log _{10} \sigma-2.303 \log _{10} \omega\right] \times 10^{-7}$

\section{Henries/metre}

which is probably the most convenient form for practical use.

It is enteresting to compare these results with thos reported by Jones et al (1961). They laid 4 different types of cable in the sea with earth returns. The cables all had steel armouring, were laid from the shore, had lengths varying from $2.96 \mathrm{~nm}$ to 9.36 nif and the far ends were in depths varying from about $20 \mathrm{~m}$ to $45 \mathrm{~m}$. The impedances were measured over the audio-frequency range and the components due to the return path calculated. Below $200 \mathrm{~Hz}$ the return path was effectively in the sea water only (that is, only a negligible proportion in the armouring), so the impedance might be expected to agree with the above calculations. They found that the resistive component was proportional to frequency and independent of cable diameter, which is in qualitative agreement, but at $200 \mathrm{~Hz}$ it was approximately $1.95 \Omega$ per nautical mile, whereas equation (14) would predict $0.365 \Omega /$ nil.

I must admit that this discrepancy is larger than I am happy with, but one might expect a factor of between 2 and 3 because the cable was on the sea-bed, whish is an insulator compared with sea water, and a proportion of the cable (near-shore) was in water shallow compared with $r(\cong 12.7 \mathrm{~m}$ at $200 \mathrm{~Hz})$. Thus, the return current was concentrated by a factor of greater than 2 compared with an infinite sea surrounding the cable.

Comprehensive tests from which full results were calculated were only made on cables 1 and 4 , respectively 4.52 and 3.41 nli long ending in water approximate1y 25 and $20 \mathrm{~m}$ deep. Cable 3 was 9.36 nif long ending in water approximately $45 \mathrm{~m}$ deep, and it may be significant that from the restricted tests made on this cable, the resistive component of the return path appears to be considerably lower.

The inductive component per nautical mile at low frequencies was found to vary by approxinately $-0.85 \mathrm{mH}$ per octave increase in frequency compared with $-0.12 \mathrm{mH}$ per octave predicted from equation (15), but at $100 \mathrm{~Hz}$ the absolute value of approximately $2.45 \mathrm{mH} / \mathrm{nm}$ for cable 4 was fairly close to the value of $2.81 \mathrm{mH} / \mathrm{n}$ il predicted from equation (15).

It is also interesting to note in passing that though the inductive component is dependent on the conductive, it is only a weak dependence and could not be used as a useful measure of it. 


\section{REFERENCES}

ANON, 1968 "Underwater wireless" Oranance. Vo1.53, p.224

(BRAINCON CORP.) 1969 "SEDAR; submerged electrode detection and ranging". Undersea Technology, Vo1.1C, p.43.

BROWN, N.L., 1968 "An in situ salinometer for use in the deep ocean". Miarine Sciences Instrumentation, Vo1.4 (F.A1t, Ed.) Plenum Press (New Yoris) pp. 563-577.

CARSON, J.R. and GILBERT, J.J., 1921 "Transmission characteristics of the submarine cable". Journal of the Franklin Institute, Vo1.192, pp. 705-735.

HORVAT, V., 1969 "Underwater radio-wave transmission". Handbook of Ocean and Underwater Engineering, Myers, J.J., Holn, C.H. and MCA11ister, R.F. (Eds.) McGraw-Hi11 (New York) pages $3-36$ to $3-40$.

JONES, F. and others (1961) "The Voice Frequency transmission characteristics of certain types of submarine telephone cables". G.P.O. Engg. Dept. Research Report No. 20202.

OLVER, F.W.J., 1964 "Bessel functions of integer order". Chapter 9 in Handbook of Hathematical Functions, fbranowitz, M. and Stegun, I.A. (Eds.). National Bureau of Standards, U.S.A.

SCHULTZ, C.W., 1971 "Underwater communication using return current density". Proc. I.E.E.E. Vo1.59, pp. 1025-1026. 


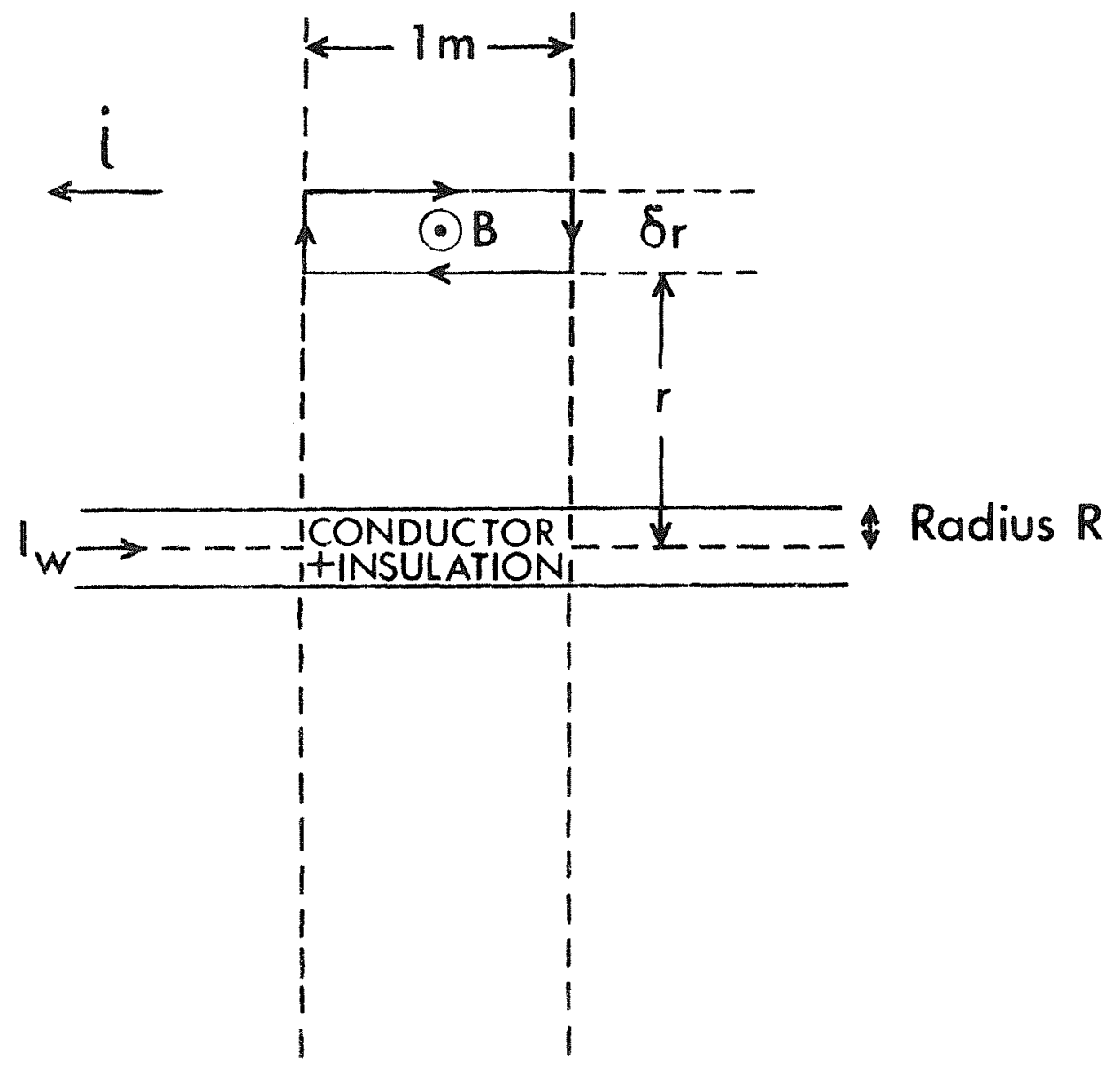

Figure 1 


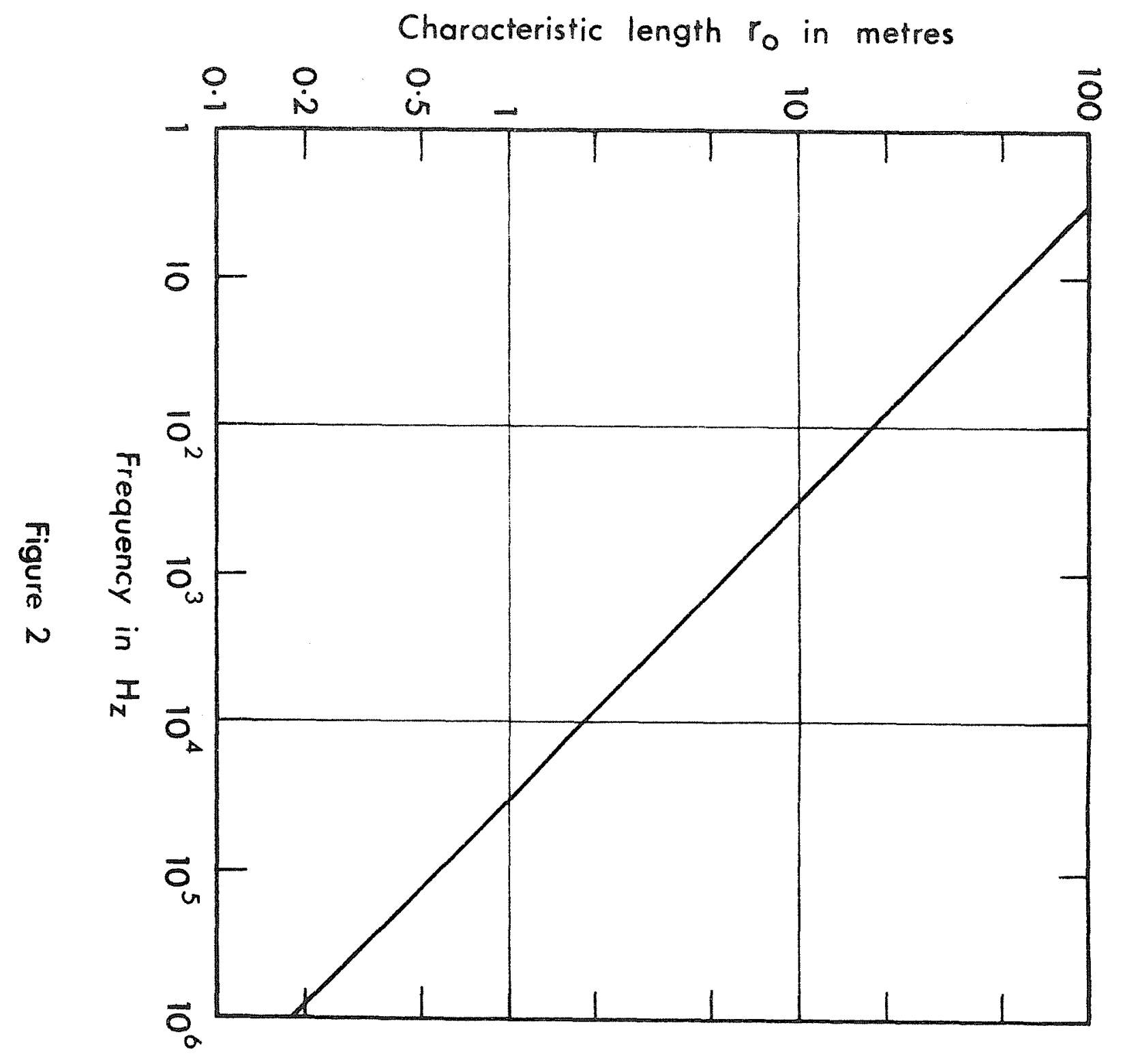




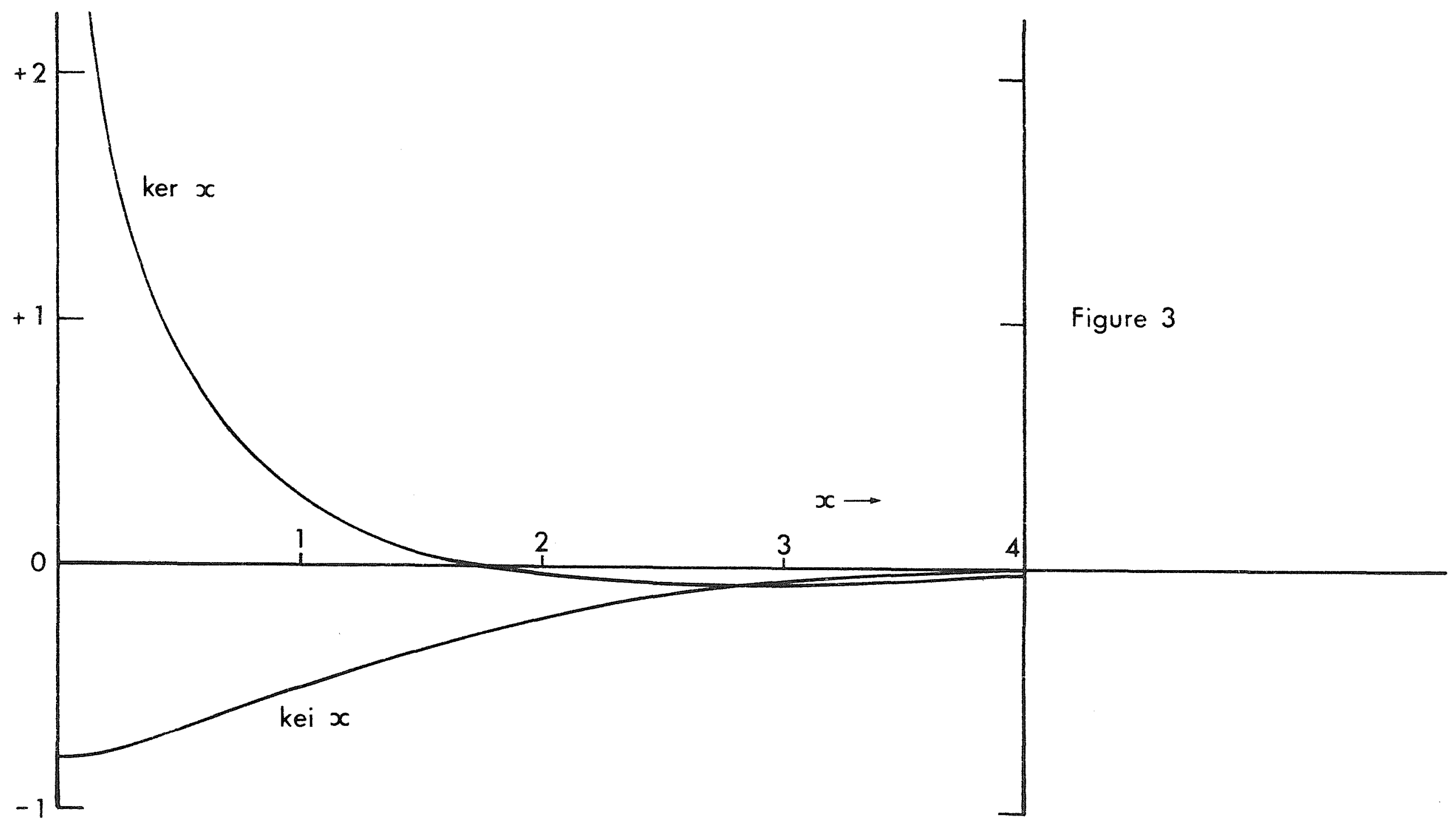




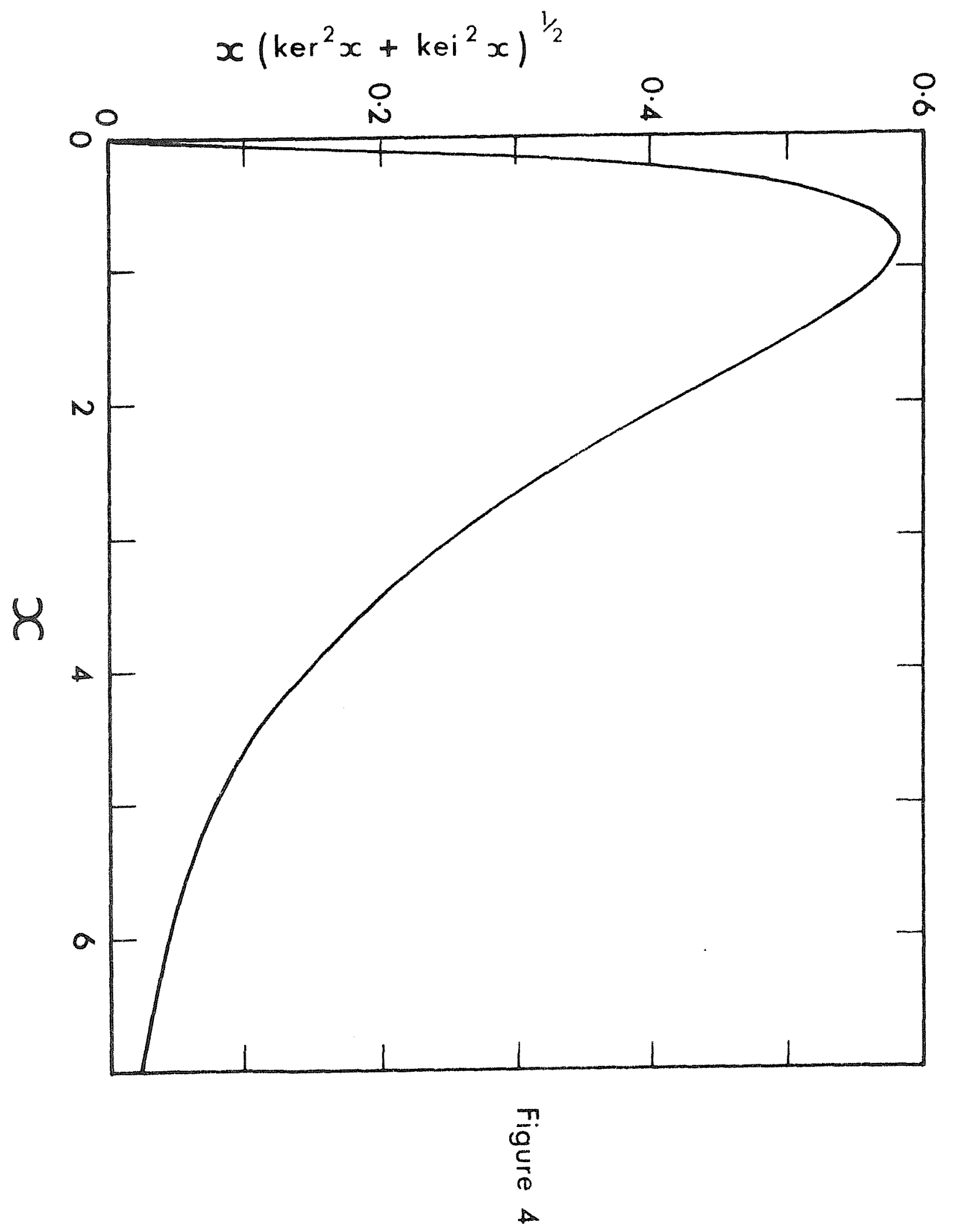


